\title{
Signaling and affective functions in Pavlovian conditioning*
}

\author{
H. FOWLER $\dagger$, G. C. FAGO $\dagger \dagger$, E. A. DOMBER $\dagger \uparrow \dagger$, and M. HOCHHAUSER \\ University of Pittsburgh, Pittsburgh, Pennsylvania 15213
}

\begin{abstract}
The present study employed a Pavlovian-instrumental-transfer paradigm to investigate the role of conditioned fear in appetitive discrimination learning. Each of three Pavlovian training procedures was used to establish a conditioned fear excitor (CS+), a "neutral" CS (CSo), and a conditioned fear inhibitor (CS-). Then, the CSs were administered to rats in the three groups contingent upon the rewarded response in a difficult visual discrimination. In addition, half of each group received shock punishment for each incorrect response. Relative to CSo, CS+ facilitated performance in contrast to the usual interfering effect of conditioned suppressors; conversely, CS - retarded performance even when its reinforcing action (fear inhibition) was potentiated by punishment for the incorrect response. These results, together with other findings showing a reversed outcome when the CSs are administered for the incorrect response, indicate that Pavlovian conditioning comprises both general signaling and affective functions, the former reflecting a basic "expectancy" or nominal type of cognitive processing in the rat.
\end{abstract}

Although the present study focused on transfer between Pavlovian conditioning and instrumental learning, it was a direct outgrowth of previous research on shock-right facilitation, i.c., the facilitating effect of mild shock for the food-rewarded response in a visual discrimination task. This paradoxical effect of punishment has been attributed to the discriminability, or cue function, of the shock (Fowler, 1971; Fowler, Fago, \& Wischner, 1971; Fowler \& Wischner, 1969). When the stimulus compounds constituting the discrimination alternatives (e.g., $T$ arms) are made similar, expectancy of reward conditioned to the cues in the rewarded arm will generalize to the cues in the nonrewarded arm (and similarly, nonreward expectancy will generalize to the rewarded arm), with the result that performance is retarded. However, when a stimulus, even an aversive one such as shock, is presented in relation to the rewarded response, it can function as a "distinctive" cue to reduce between-arm generalization effects and thus facilitate discrimination-given, of course, that the cue effect of the shock is sufficient to overcome its aversive effect.

Two investigations have been designed to separate the cue and aversive components of punishment. In the first of these (Fowler, Goldman, \& Wischner, 1968), amobarbilal was used to reduce the aversive effect of different intensities of shock administered for the rewarded response in a difficult bright-dim discrimination. The results of this study showed that in

*This study was supported in part by Grant MH-08482 from the National Institute of Mental Health, United States Public Health Service, and by Grant GB-24119 from the National Science fioundation.

†Requests for reprints should be sent to Harry Fowler, Department of Psychology, University of Pittsburgh. Pittsburgh, Pennsylvania 15213.

$\dagger \uparrow$ Now at Ursinus College, Collegeville, Pennsylvania 19426. $+\dagger \dagger$ Now at Drew University, Madison, New Jersey 07940. contrast to no-drug controls, for which performance was initially facilitated and then retarded across increasing intensities of shock, the discriminative performance of Ss injected with amobarbital improved as an S-shaped function of shock intensity, consistent with the Weber function relating performance to the discriminable cue properties of a stimulus. In a recent extension of this work, Fago and Fowler (1972) investigated a distinctive-cue effect of aversive stimulation by varying the intensity of response-contingent white noise from sub- to supraaversion values. With this manipulation, it was possible to assess the cue effect of white noise at weak (nonaversive) intensities and the interaction of the cue and aversive components at higher (aversive) intensities. Consistent with the earlier findings, this study has shown that performance in a difficult bright-dim discrimination is comparably facilitated by noise administered for either rewarded or nonrewarded responses, indicating that the effect is independent of a specific avoidance-producing function of the noise stimulus. Further, such facilitation was an S-shaped function of noise intensity, again consistent with the Weber function.

Paralleling these prior efforts, the Fago and Fowler (1972) study provides the means by which the avoidance-producing (e.g., fear) component of an aversive stimulus can itself be isolated not merely to demonstrate avoidance, but rather to assess the possible role of fear and its feedback as a distinctive stimulus event. Given the S-shaped relationship between the facilitating cuc effect of noise and noise intensity, one can use Pavlovian training to establish a weak noise stimulus (with minimal cue value and no inherent aversiveness) as a conditioned fear excitor, i.e., as a stimulus which has, so to speak, "acquired distinctiveness" through conditioned fear and its feedback component (cf. Lawrence, 1949, 1950). Following this procedure, the present study generated a 
"fear-right" training paradigm by presenting a conditioned aversive stimulus for food-rewarded responses in a difficult bright-dim discrimination. To control for the CS and US (shock) experiences of Pavlovian training, a "neutral" CSo condition was included in which Ss received CS and US presentations in an uncorrelated fashion (cf. Rescorla, 1967). To control for the specific type of reaction (and related feedback) conditioned during Pavlovian training, a CScondition was employed in which Ss received backward presentations of CS and US, i.e., a negative correlation of CS and US (cf. Rescorla, 1969). Contrasting with the forward conditioning procedure used to establish the conditioned fear excitor (CS+), the backward procedure effectively establishes a conditioned fear inhibitor (CS-) for which the Pavlovian reaction may be viewed as conditioned "relief" and/or "relaxation" (cf. Denny, 1971; LoLordo, 1969). Finally, to control for any distinctive-cue function of the weak noise CS (resulting possibly from a sensitization effect), a group comprising Ss from all three CS conditions received no CS during appetitive discrimination training.

In discrimination training, the CSs were presented for the food-rewarded response so as to generate "signal-right" training analogous to shock-right and noise-right training. In addition, half of the Ss of every group inclusive of the no-CS control received shock for the incorrect (nonrewarded) response. This "shock-wrong" condition was included for two reasons: first, to generate a fearful discrimination context and thereby potentiate for CS- Ss a conditioned fear-inhibition (reinforcement) effect in the food-rewarded arm and in this manner facilitate performance; second, to promote for CS+ Ss an "added equivalence" effect by producing fear in both rewarded and nonrewarded arms (as a result of the CS+ and shock, respectively) and in this manner degrade performance. Basically, then, the study entailed a 2 by 4 factorial design of no-shock and shock-wrong discrimination training and three types of Pavlovian signals (CS+, CSo, CS-) for the rewarded response, plus a no-CS control. When the initial findings of the study were apparent, however, two additional Pavlovian procedures (differential between-Ss and conditioned-inhibition training) were added to confirm the CS- (as well as the CSt) effects. Hence, the study was extended to a 2 by 4 by 3 factorial design in which the last factor constituted three different Pavlovian procedures.

\section{METHOD}

\section{Subjects}

The Ss were 96 male albino rats of the Sprague-Dawley strain, purchased from the Holtzman Co. at about 90 days of age. The Ss were individually caged in both the experimental room and an adjacent sound-attenuated room where the temperature was controlled at $68^{\circ}-72^{\circ} \mathrm{F}$ and the day-night cycle reversed through artificial illumination.

\section{Apparatus}

The Pavlovian-conditioning apparatus was a black wooden chamber $(26.7 \times 19.0 \times 15.2 \mathrm{~cm}$ deep) with a clear Plexiglas top and a stainless steel grid floor. A Jensen (Model P4V3) $10.2-\mathrm{cm}$ 3.2-ohm speaker was centered on top of the Plexiglas lid and was connected through a selector switch and timer circuitry to the output of both a Grason-Stadler (Model 901B) white-noise generator and a Hewlett-Packard (Model 200CD) wide-range oscillator. These signal generators provided the CS: a 70-dB $0.2-\mathrm{sec}$ burst of white noise or a $200-$ or $2,000-\mathrm{Hz}$ tone of equal intensity and duration. Intensity determinations were made by a General Radio Type $1565 \mathrm{~A}$ sound meter using a C-scale setting against an ambient noise level of about $60-65 \mathrm{~dB}$. The grid floor of the chamber, comprising 23.8-mm-diam rods spaced $1.59 \mathrm{~cm}$ apart, was connected through timer circuitry and a series resistor of 0.24 megohms to the output of a variable transformer. This fixed-impedance shock source provided the US: a $60-\mathrm{Hz}$ ac shock of $0.2 \mathrm{sec}$ duration and $120 \mathrm{~V}$ as measured across the transformer output (essentially $0.5 \mathrm{~mA}$ to the grid).

The discrimination-training apparatus was an enclosed T-maze consisting of a black wooden shell and a clear Plexiglas top. The width and height of the maze were uniformly 9.53 and $11.4 \mathrm{~cm}$, respectively, with the length of the stem being $66.0 \mathrm{~cm}$ and each arm $91.4 \mathrm{~cm}$. The internal structure of each maze section was formed of two L-shaped strips of galvanized sheet metal, each L serving as a wall and half of the floor. Together, the two L-shaped strips provided two $3.8-\mathrm{cm}$ floor surfaces that were separated by a $1.9-\mathrm{cm}$ gap. Guillotine doors were located at the entrance to each arm, $7.6 \mathrm{~cm}$ from the center of the choice point, $20.3 \mathrm{~cm}$ from the near end of the stem to form a start compartment, and $30.5 \mathrm{~cm}$ from the end of each arm to form the goal compartments. Each goal compartment had a clear Plexiglas foodwell $(1.9 \mathrm{~cm}$ in diam $\times 1.3 \mathrm{~cm}$ deep) that was accessible at the base of the floor between the two L strips and a frosted Plexiglas end plate that served as the discriminative stimulus when it was illuminated from behind by a $15-\mathrm{W}$ incandescent bulb enclosed in a wooden case. To effect a difficult bright-dim discrimination, the goalbox bulbs were operated at 120 and $62.5 \mathrm{~V}$. Ambient illumination was provided by two partially shielded $15-\mathrm{W} 120-\mathrm{V}$ bulbs located exterior to the maze on either side of the stem.

To effect CS presentation in discrimination training, identical Jensen (Model P4V3) 10.2-cm 3.2-ohm speakers were mounted on the transparent Plexiglas tops of the maze arms, $10.2 \mathrm{~cm}$ from the goalbox doors. Each speaker was independently connected through switching and timer circuitry to the output of both the Grason-Stadler white-noise generator and the Hewlett-Packard tone oscillator and was activated by the interruption of an infrared photoelectric beam crossing the arm at a point directly beneath the speaker. Thus, a particular CS (tone or noise) could be delivered in either arm. A manual priming feature of the CS circuitry prevented $S$ from receiving more than one $\mathrm{CS}$ presentation per trial. When the CS was delivered, its intensity and duration were identical to those values ( $70 \mathrm{~dB}, 0.2 \mathrm{sec}$ ) employed in Pavlovian training; similarly, ambient noise within the T-maze was approximately $60-65 \mathrm{~dB}$.

A fixed-impedance shock system consisting of a $60-\mathrm{Hz}$ ac source and a series resistance of 0.24 megohms was used to deliver shock to $S$ in either maze arm. With this shock system, the two L-shaped strips of sheet metal forming the walls and floor of the maze were connected across the transformer output; hence, $\mathrm{S}$ received shock when it made contact with both halves of the sheet metal floor. Due to the narrowness of the maze alley, $S$ could not avoid shock by running or standing on only one half of the floor. Shock was delivered to $S$ when $S$ interrupted, in either arm, the infrared photobeam located directly beneath the CS speaker. A manual priming feature of the shock circuit prevented $S$ from receiving more than one shock per trial. When shock was delivered, its duration was held constant at $0.2 \mathrm{sec}$ and its intensity set at $60 \mathrm{~V}$ as measured 
across the transformer output (essentially $0.25 \mathrm{~mA}$ to the maze floor).

\section{Procedure}

The experimental procedure consisted of three stages of training conducted in the following order: nondifferential pretraining in the T-maze, Pavlovian conditioning in the chamber. and appetitively reinforced discrimination training in the T-maze. One weck prior to pretraining. Ss weighing $300 \mathrm{~g}$ or more were started and maintained for the duration of the experiment on a daily diet of $11 \mathrm{~g}$ of Wayne Lab Blox with water available ad lib. Pretraining was conducted to habituate $S$ to the T-maze and to reduce possible position and brightness preferences. Each $S$ received a total of 16 forced-choice food-rewarded trials administered 4 per day at an intertrial interval (ITI) of about 6-8 min and randomly distributed with the restriction that $S$ 's daily trials were balanced over both right and left arms and bright and $\operatorname{dim}$ goals. Iorcing was accomplished by lowering the guillotine door at the entrance to the inappropriate arm. On the first pretraining day, food reward (P. J. Noyes Formula $A$ rat pellets. $4 \mathrm{~mm} .45 \mathrm{mg}$ ) was liberally spread throughout a goal compartment and then. on succeeding day's, systematically reduced, until on the last day only two pellets were administered in the goalbox foodcup.

Pavlovian training began on the day following the completion of pretraining and consisted of three different training procedures for different thirds of the Ss: (a) forward-backward, (b) differential between-Ss. and (c) conditioned-inhibition training. As noted, assignment of the $S s$ to the three procedures was not random within a sample, but constituted successive replications of $S s$ in the order designated. With all three procedures. Ss received 6 consecutive days of training entailing a daily series of discrete Pavlovian trials administered at an ITI of about 6-8 min. When presented, the CS and US events occurred only once on any trial. Between trials, the Ss were placed in holding cages which were insulated from sounds (CS presentations) emanating from the Pavlovian chamber. With each Pavlovian procedure, the $S$ s were randomly assigned (with restrictions detailed below) to three different training regimes designed to establish the particular CS (noise or tone) as a conditioned fear excitor ( $\mathrm{CS}+$ ), a conditioned lear inhibitor (CS -), or as a nonfunctional CS (CSo). Following Rescorla (1967. 1969), the operational rule for establishing the particular type of $\mathrm{CS}(+,-.0)$ within each procedure was as follows: CS + , positively correlated with the occurrence of the US in a "forward" order. i.e.. IS contingent upon the CS; CS -. negatively correlated with the occurrence of the US, i.e., US contingent upon the absence of the CS; CSo, uncorrelated with the occurrence of the US. i.e., no contingency. These three correlations, or contingencies. may be expressed in the form of conditional probabilitics where the signal value (v) of the CS is described by $\mathrm{p}(\mathrm{US} / \mathrm{CS})$ - $\mathrm{p}(\mathrm{US} / \overline{\mathrm{CS}})$ : hence, $v(\mathrm{CS}+)=1.0-0.0=$ +1.0 : $v(\mathrm{CS}-)=0.0-1.0=1.0$; and $v(\mathrm{CSO})=0.5-0.5=0.0$.

The specifics of each Pavlovian procedure were as follows. (a) Forward-backward training: Ss received $860-\mathrm{sec}$ trials per day for a total of 48 trials. On all trials, the CS (noise) occurred in random 5-sec steps at $15-45 \mathrm{sec}$ into the trial. For CS+ Ss, the US always followed the CS by $2.0 \mathrm{sec}$, whereas for CS -- Ss, the US always preceded the CS by $2.0 \mathrm{sec}$. For CSo Ss, the US occurred independently of the CS in random 5-sec steps at $12.5-47.5 \mathrm{sec}$ into the trial, but with the restriction that on half of the trials the US followed the CS and on the other half it preceded the CS (cf. "random" control; Rescorla, 1967). (b) Differential between-Ss training: Ss received 16 30-sec trials per day for a total of 96 trials. On a random half of these trials, the US occurred in random 5-sec steps at $5-25 \mathrm{sec}$ into the trial. For CS+ Ss, the CS (noise) was presented only on US trials $2.0 \mathrm{sec}$ prior to the US: on the other half of the trials, neither the CS nor the US was presented. For CS - Ss, the CS occurred only on non-LS trials, at times identical to its occurrence for the
$\mathrm{CS}+$ Ss. For CSo Ss, half of the trials were of the CS+ variety (noise + shock and neither event) and half were of the CSvariety (shock alone and noise alone) to effect a "balanced" control. (c) Conditioned-inhibition training: Ss similarly received 1630 -sec trials per day for a total of 96 trials. On all trials, one CS (either a 200 - or $2,000-\mathrm{Hz}$ tone) occurred in random $5-\mathrm{sec}$ steps at 3-23 sec into the trial. For $C S+/ C S-S s$ (a within-S manipulation), the US followed the CS by $2.0 \mathrm{sec}$ on a random half of the trials: on the other half of the trials, the second CS (the other of the two tones. counterbalanced across Ss) followed the first CS by $1.5 \mathrm{sec}$ without the LS being presented. Thus, the first $C S$ by itself was always positively correlated with the occurrence of the US and served as a CS+, whereas the second CS was always negatively correlated with the occurrence of the US and served as a CS-. For CSo Ss, a random half of the trials were of the same type (Tone $1+$ shock and Tone $1+$ Tone 2 without shock), whereas the other half of the trials obviated the Tone 1 and Tone 2 correlations with shock by reversing them, i.e., presenting Tone 1 alone without shock and Tone $1+$ Tone 2 with shock (same ISIs) on an equal number of trials, again to produce a "balanced" control.

Discrimination training in the $T$-maze began on the day following the completion of Pavlovian training and consisted entirely of free-choice noncorrection trials. Trials were administered four per day for the first 6 days and eight per day thereafter at an ITI of about 6-8 min. As in Pavlovian training, Ss were placed in the sound-insulated holding cages during the ITI On each trial. food reward at two pellets $(45 \mathrm{mg}$ each) was available only in the brighter goal, the left-right position of which varied randomly across trials. On each trial, detention time in either the correct or incorrect goal was approximately $10 \mathrm{sec}$. Different subgroups of $\mathrm{Ss}(\mathrm{N}=8)$ from each of the three Pavlovian-conditioning procedures received a $\mathrm{CS}+, \mathrm{CSo}, \mathrm{CS}-$, or no CS contingent upon each correct response. The no-CS condition served as a general control for possible distinctive-cue properties of the noise and tone CSs and comprised, across the three Pavlovian conditions and to the extent possible within each Pavlovian procedure, an equal number of $\mathrm{CS}+, \mathrm{CS}-$, and CSo Pavlovian-trained Ss. (Specifically, the numbers of CS+, CSo, and CS-Ss within the no-CS condition were, respectively, 3,2, and 3 from the forward-backward procedure, 3,2 , and 3 from the differential procedure, and 2,4 , and 2 from the conditioned-inhibition procedure. Because the latter was a within-S procedure, $C S+/ C S$ - Ss were arbitrarily designated as one type or the other for inclusion in the no-CS condition.) To potentiate a conditioned fear-inhibition (CS-) effect in discrimination training, as well as to promote an "added equivalence" effect within the CS+ condition, as previously described, half of the Ss of each CS group, including the no-CS control, received shock punishment for each incorrect response (shock-wrong training), whereas the other half received no shock for either correct or incorrect responses (no-shock training). Collectively, then. there were 24 groups of four Ss each comprising a 2 by 4 by 3 factorial design of no-shock and shock-wrong discrimination training, both of which entailed the administration of an aversive CS+, CSo, CS . or no CS for each rewarded response, wherein the different CSs had been established by forward-backward, differential, or conditioned-inhibition Pavlovian training. Discrimination training was conducted until each $S$ reached a criterion of 15 correct choices on 16 consecutive trials with the last 8 correct. or until a total of 300 trials had been administered.

\section{RESULTS}

Group mean errors in blocks of 20 trials are presented for Ss of the CS+. CSo, CS--, and no-CS conditions in Fig. 1. (Subgroups of Ss representing the three Pavlovian procedures and the no-shock and shock-wrong 


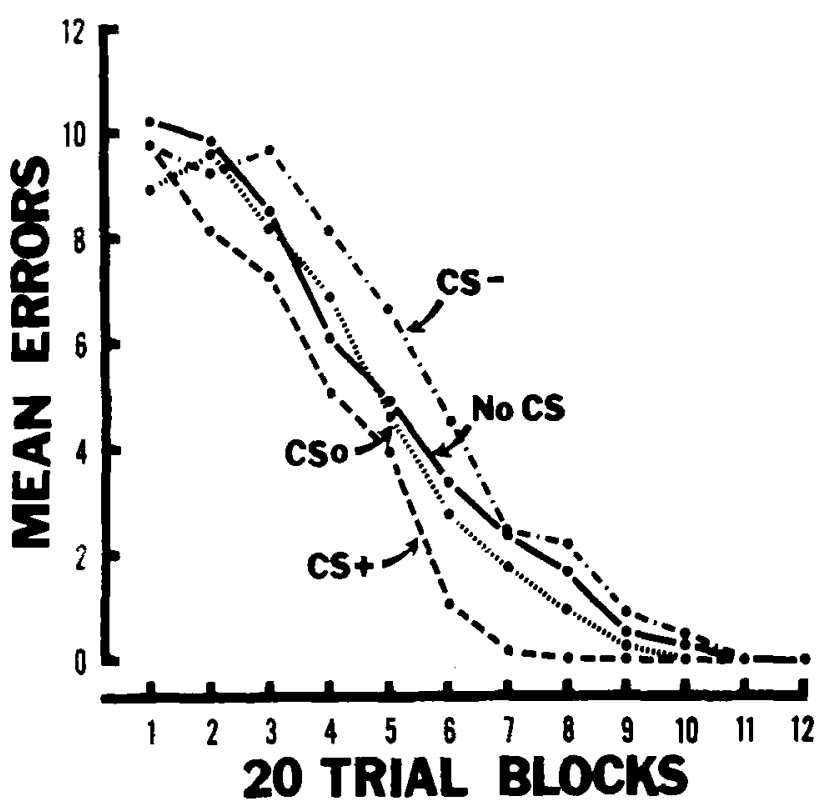

Fig. 1. Group mean errors in blocks of 20 trials for Ss of the $\mathrm{CS}+$, CSo, CS-, and no-CS conditions. (Subgroups of Ss representing the three Pavlovian procedures and the no-shock and shock-wrong discrimination-training conditions have been pooled.)

discrimination-training conditions have been pooled in order to reduce the cluster of curves.) As shown. errors were eliminated at virtually the same rate under the CSo and no-CS conditions, indicating that the weak $70-\mathrm{dB} C S$ (noise or tone) had no facilitating effect on performance per se, i.e., as a distinctive cue. Consistent with our general expectations, the aversive CS+ ("fear-right") condition produced a more rapid elimination of errors relative to the CSo condition, but contrary to our expectations, the aversive CS - ("relief-right") condition produced a comparably slower elimination of errors. Accordingly, the results of an analysis of variance of errors to criterion showed that the CS main effect was highly reliable $[F(3,72)=5.32, p<.005]$. Furthermore, polynomial comparisons within the analysis of variance indicated that the difference between the CSo and no-CS conditions was negligible $(\mathrm{F}<1)$, but that the linear trend across CS effects (i.e., CS+ through CSo and no CS combined to $\mathrm{CS}-$ ) was highly significant $[\mathrm{F}(1,72)=$ 15.27. $p<.001]$ and accounted for virtually all $(96 \%)$ of the variance due to the CS factor. Given that residual-trend variance after extraction of this linear component was negligible $(F<1)$, the best description of these data is that errors to criterion were a linear function of CS sign (i.e., CS-US correlation: +1.0 through 0.0 to -1.0 ); hence, the facilitation and retardation effects for the CS+ and CS-conditions were symmetrical about their reference (CSo) condition and of equal magnitude. The appropriateness of this description becomes evident when it is considered that the negligible $\mathrm{F}$ ratio for residual-trend (i.e., quadratic) variance derives from a comparison of the $\mathrm{CS}+$ and $\mathrm{CS}$ - groups with the CSo and no-CS groups combined. Thus, consistent with their signal values, the sum of the error scores for the functional CS groups $(+1.0$ and -1.0$)$ yielded an outcome equal to that for the nonfunctional and no-CS groups $(0.0)$.

The linear ordering of CS effects is illustrated more clearly in Fig. 2, which presents mean errors to criterion as a function of CS-US correlation for Ss grouped according to the three Pavlovian procedures (left panel) and according to the no-shock (NS) and shock-wrong (SW) discrimination-training conditions (right panel). Within each panel, respective no-CS groups are indicated by corresponding data points with horizontal blips. As shown in the left panel, the linear CS effect was common to all three Pavlovian procedures with differences among the procedures being minimal and unsystematic. Thus. the $F$ test results showed that the effect of Pavlovian-training procedure was nonsignificant $[F(2,72)=0.67]$. as well as all comparisons among the procedures ( $p s>.20)$, and further that the interaction of Pavlovian procedure with $\mathrm{CS}$ sign (+, o. --) was nonsignificant $[F(6.72)<1]$. including all components of the interaction. These nonsignificant effects were not due to the inclusion of the no-CS groups; analysis of the data for Pavlovian procedures with the no-CS groups excluded also yielded negligible effects $(\mathrm{F}<1)$. Indeed, the only apparent aberration in the data is that relating to the no.CS and CSo groups of the forward-backward procedure. Even this difference, however, when assessed by polynomial comparisons either directly or as part of the Procedure by $C S$ interaction. proved unreliable $(F s<1)$. Thus. despit seemingly complex differences in the nature of the three procedures. particularly with respect to the establishment of a $\mathrm{CS}-$. all three

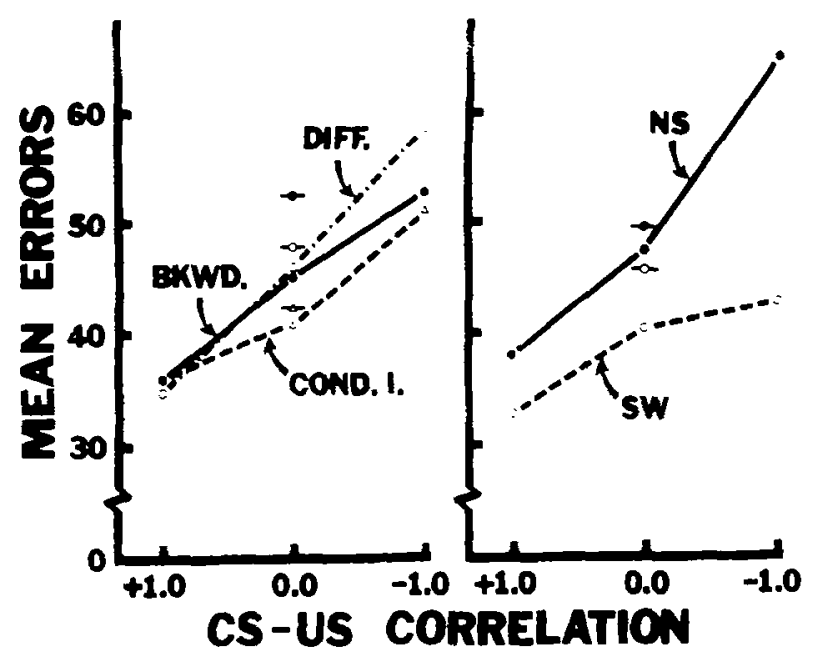

Fig. 2. Mean errors to criterion as a function of CS-LS correlation for Ss grouped according to the three Pavlovian-training procedures (left panel) and according to the no-shock (NS) and shock-wrong (SW) discrimination-training conditions (right panel). Respective no-CS controls are represented by corresponding data points with horizontal blips. 
procedures generated comparably facilitated performance for the $\mathrm{CS}+$ groups and comparably retarded performance for the CS-- groups. When it is further considered that the three Pavlovian procedures constituted independent replications and were subject to this added source of variance, the absence of differences among the three procedures is particularly striking and may attest to the robustness of the CSt and CS effects.

The right panel of Fig. 2 shows that the linear relationship between errors and CS-US correlation was present as well for Ss of the NS and SW discrimination-training conditions but that the $\mathrm{SW}$ treatment had the effect both of reducing errors. consistent with its suppressing and/or distinctive-cue functions, and to some extent of reducing the slope of the linear CS effect. Accordingly, the F test results showed that the shock main effect was significant $[F(1,72)=8.19, \mathrm{p}<.01]$ and that the linear component of the Shock by CS interaction was at least marginally reliable $[F(1,72)=3.43, p<.08]$, suggesting that the magnitude of the difference between respective NS and $\mathrm{SW}$ groups increased from $\mathrm{CS}+$ to $\mathrm{CS}-$. To further assess this interaction, polynomial comparisons were performed on the error means for respective NS and SW groups of the CS+ and $\mathrm{CS}-$ conditions. These comparisons, as evaluated against the overall error term, showed that the difference between NS and SW groups of the CS+ condition was negligible $(F<1)$, whereas that for the NS and SW groups of the CS- condition was highly reliable $[F(1,72)=11.37, p<.005]$. All other comparisons pertaining to the Shock by CS interaction. including residual-trend components and a comparison of the no-CS and CSo groups, were nonsignificant. Similarly, the interaction of shock treatment with Pavlovian procedure and the second-order interaction of shock with Pavlovian procedure and CS sign were also nonsignificant.

Given the significant shock main effect and the negligible effect of Pavlovian procedure, a separate analysis of variance was performed on the error scores for the three CS $(+, 0,-)$ subgroups of the no-CS condition with variance due to the shock treatment extracted. The results of this analysis were also nonsignificant $(F<1)$, indicating that prior type of CS training had no effect on the discrimination performance of $S s$ in the no-CS condition.

Figure 3 presents mean trials to criterion as a function of CS-US correlation for Ss grouped according to the three Pavlovian procedures (left panel) and according to the NS and SW discrimination-training conditions (right panel). Again, respective no-CS groups are indicated by corresponding data points with horizontal blips. The data on trials to criterion were virtually identical to those on errors. There was a reliable CS main effect $[F(3,72)=6.19, \mathrm{p}<.001]$, with a highly significant linear component $[F(1,72)=18.14, p<.001]$ and negligible residual-trend effects inciuding a nonsignificant difference between the no-CS and CSo

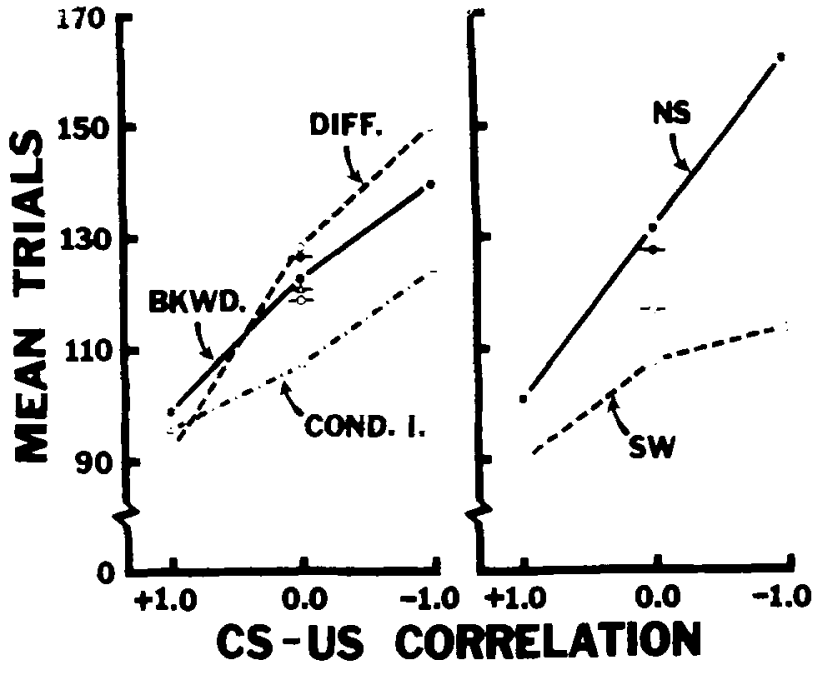

Fig. 3. Mean trials to criterion as a function of CS-US correlation for Ss grouped according to the three Pavlovian-training procedures (left panel) and according to the no-shock (NS) and shock-wrong (SW) discrimination-training conditions (right panel). Respective no-CS controls are represented by corresponding data points with horizontal blips.

groups (Fs $<1$ ). The main effect of Pavlovian procedure was again unreliable (both with and without inclusion of the no-CS groups, $p>.20$ in both cases), including all specific comparisons among the procedures ( $p>.10$ in all cases), and the interaction of Pavlovian Procedure by CS Sign was also nonsignificant $(F<1)$. In comparable fashion, the SW treatment reduced both trials $[F(1,72)=$ $11.30, p<.005]$ and to some extent the slope of the linear CS effect, the latter as indicated by a marginally reliable linear component of the Shock by CS interaction $[F(1,72)=3.50, p=.07]$. That the difference between respective NS and $\mathrm{SW}$ groups increased from the $\mathrm{CS}+$ to the $\mathrm{CS}-$ condition was again indicated by a negligible difference between those groups of the CSt condition $(F<1)$ and a highly reliable difference between those of the CS- condition $[F(1,72)=11.93, p<.0011$. These data, in matching those on errors, are important in showing that the linear CS effect was not diminished but in actuality was amplified by the use of a measure sensitive as well to correct responses for which the aversive CSs were contingent. Indeed, subtraction of the error means from the trial means yields virtually identical functions for correct responses and reflects the similarity of effect of CS treatment on both rewarded and nonrewarded responses.

\section{DISCUSSION}

\section{Procedural Variations in Pavlovian Training}

An interpretation of the present results is simplified somewhat because of the absence of differences relating to certain factors operating in the three Pavlovian procedures. These factor dimensions fall into three 
categories: (a) variations in $\mathrm{CS}-$, (b) variations in CSo. and (c) their relation to the development of chronic and acute fear. As previously indicated, the differential between-Ss and conditioned-inhibition procedures were added to the experiment primarily to confirm the retardation effect initially observed for the CScondition of the forward-backward procedure. While all three procedures employed a negative correlation of CS and US to establish the conditioned fear inhibitor (CS-), they differed markedly in the specific procedural arrangements by which this was accomplished, i.e., through presentation of the CS after the US (backward). in the absence of the US (differential), or in place of the US following the CSt (conditioned inhibition). The absence of differences in outcome among the three procedures strongly implicates the negative correlation or contingency of CS and US as the factor common to the observed CS- retardation effect and is consistent with Rescorla's (1969) conclusion that the three types of procedures yield comparable conditioned-inhibition effects. Furthermore, because a negative correlation of CS and US is effectively employed as half of the CSo training condition (to offset that half entailing a positive correlation of CS and US), the absence of differences among the three procedures also implicates the noncontingency, or zero correlation, of CS and US as the factor common to the observed CSo effects.

With regard to the CSo condition in particular, the three procedures differed in their use of either a "truly random" procedure (forward-backward training) or a "balanced" control procedure (differential and conditioned-inhibition training). In a truly random procedure, a zero correlation of CS and US events is effected by scheduling these events independently of one another over the course of a trial or session. Thus, in contrast to CSt and CS- conditions (e.g., forward and backward training, respectively) for which the CS and US occupy a specific temporal relationship to one another (ISI), as well as a specific point in time on any trial, the truly random procedure not only obviates a correlation of CS and US events, but in addition varies the ISI as well as the temporal loci of CS and US within a trial, and possibly also their frequency, given that the scheduling of events is completely random. These nonassociative confoundings are ruled out, however. with the use of a "balanced" control procedure where CSo is established by randomizing the order but balancing the number of CSt and CS-trials so that the CS and US events on a trial occupy the same temporal relationships and points in time as exist for Ss trained on the CSt and CS - conditions (cf. differential procedure). Given these potentially important differences, the failure of the three procedures to produce differences for the CSo groups may reflect the absence of such nonassociative effects and point to the zero contingency or correlation of CS and US as the factor singularly responsible for the intermediate performance outcome generated by the CSo condition.
The three Pavlovian procedures also differed in their use of either a between-Ss design (forward-backward and differential training) or a within-S design (conditioned-inhibition training) to establish a particular type of CS. These differences underlie the potential development of chronic and acute states of fear for the different CS conditions of the three Pavlovian procedures (see Seligman, 1969; Seligman, Maier, \& Solomon, 1971). For example, in the between-Ss design (e.g., differential), a CS - is formed by presenting a discrete CS (e.g., noise) in the absence of the US and, likewise, the US in the absence of the CS. Under these conditions, static apparatus (e.g.. background) cues are positively correlated with shock and become an effective CSt. In contrast to a discrete CSt (noise) which consistently signals immediate shock and thus sets the basis for conditioning of acute fear, background cues serving as a CS+, by their static nature, cannot reliably signal immediate shock; consequently, the CS-Ss are in a chronic state of fear. Furthermore, because CStraining represents a functional half of the CSo condition, in that for $\mathrm{Ss}$ of this condition discrete stimulus events do not consistently signal immediate shock. CSo Ss of a between-Ss design are similarly exposed to chronic fear. Unlike the between-Ss design, however, the within-S design (conditioned-inhibition procedure) employs two discrete events, establishing one as a CS+ and the other as a CS-. Consequently, for Ss of this condition, immediate shock is always reliably signaled by one event and hence fear will be acute with this event. (This is the case even in the balanced CSo condition of the within-S design.) Despite these differences, all three procedures yielded virtually identical outcomes for the different CS conditions, indicating that factors such as chronic and acute fear were not determinants of the observed effects.

\section{Interpretations Based on Fear and Other Specific Responses}

Apart from differences in chronic and acute fear, the role of fear itself can be questioned as a factor influencing the obtained results. Administering a conditioned aversive stimulus (AvCS+) for the rewarded response (fear-right training) did not in any way suppress correct responding or interfere with performance generally, as would be expected on the basis of a conditioned punishment procedure; rather, AvCS+ facilitated performance. Such facilitation could be explained in a manner analogous to shock-right facilitation, that fear and its stimulus feedback served as a distinctive cue to increase the discriminability of the choice alternatives and reduce between-arm generalization effects (Fowler, 1971). However, this interpretation is weakened by the fact that a conditioned fear inhibitor (AvCS-), for which a "relief" reaction (Denny, 1971) and its feedback component should similarly serve as a distinctive cue, did not 
facilitate performance but rather retarded it, and to the same extent that AvCSt facilitated performance. Furthermore, this symmetrical relationship between AvCSt and ArCS -- was maintained with SW training, albeit at a reduced slope. According to a distinctive-cue interpretation, in SW training an AvCS+ should produce an "added equivalence" effect (as a result of fear being present in both alternatives) and thus retard performance; in contrast, an AvCS- should generate added distinctiveness as a result of the fear produced by shock in the incorrect alternative and the fear inhibition (relief) provided by the $\mathrm{CS}$ - in the correct alternative. Indeed, contrasted with the shock-produced fear in the incorrect alternative, the presence of an AvCS- in the correct alternative should have allowed additional reinforcement (fear reduction, conditioned relief) for correct responses and thus it should have had a facilitating rather than a retarding effect on performance.

Other interpretations based on the eliciting properties of the AvCSs can be entertained but equally without success. For example, an AvCS+ presented in the correct arm, eliciting a cringing or startle reaction (cf. McAllister \& McAllister, 1971), might enable $S$ in some manner to become alerted and/or attentive to the $S^{\mathrm{D}}$ in the context of impending food reward; and an AvCS- eliciting a comparable fear-releasing or noncringing reaction might interfere with $S$ 's attending to the $S^{D}$. Another possibility is that AvCS+ caused $S$ to lurch or dart forward into the correct goal. analogous to the motor-eliciting effect of shock punishment (Fowler, 1963; Fowler \& Miller, 1963), so that food reward was more immediate for CS+ than for CS-Ss, because CSpresumably produced a comparable interfering reaction and hence a delay of reward. These kinds of explanations become severely strained, however, when they are made to account not only for CS+ facilitation and $\mathrm{CS}$ - retardation, but also for the fact that these effects are symmetrical about the CSo (and no-CS) reference condition. Furthermore, it is difficult to reconcile interpretations stressing specific reactions such as cringing, lurching forward. etc.. with the comparable but reduced linear ordering of CS effects in the SW treatment. The most direct contrary evidence for such interpretations comes from a recent study (Domber, 1971) which shows that the facilitation and retardation effects produced by AvCSt and AvCS . are exactly. reversed when the CSs are administered for the incorrect response. Under these conditions (in the absence of the SW treatment). AvCS+ retards and AvCS facilitates performance, the effects again being symmetrical about a CSo reference condition. Hence, any interpretation of the effects of the CSs in terms of specific motor reactions that are classically or even operantly conditioned during Pavlovian training (see Trapold \& Overmier. 1972), and are elicited during discrimination training. must explain not only the equal and opposite effects of an AvCSt and an AvCS - administered for one response (either correct or incorrect), but also the equal and opposite effects of the same CS administered for correct and incorrect responses.

\section{Signaling and Affective Properties of Conditioned Stimuli}

Because the present results seem equally opposed to extant interpretations of the Pavlovian-instrumental interaction (see Rescorla \& Solomon, 1967), we are of the opinion that the results call for a reinterpretation of the nature of Pavlovian conditioning itself. In particular, we would suggest that Pavlovian conditioning is to be viewed as involving two kinds of learning. In the first. $S$ learns that the CS signals that an event (the US) is either coming (as with an "additive" signal, $\mathrm{CS}+$ ) or is not coming (as with a "subtractive" signal, CS-). Thus, appropriate to its defining operation, a CSo signals neither that the US is coming or is not coming. Secondly, depending upon whether the US is aversive or appetitive, the general signaling property of the CS has associated with it a particular affective value. A CS acts, then, as a signal for the presence or absence of something which is either affectively positive or affectively negative, e.g., food or shock. Our use of the term "affective value" is simply for expository purposes, for it in no way rules out specific motor and emotional (e.g., fear, relief) reactions that can be occasioned by the US or its absence and conditioned to the CS. That is to say, in signaling the occurrence or nonoccurrence of an event, the CS can, through the affective value associated with its signaled event (e.g. an aversive US), come to control those specific responses (e.g., fear and cringing or, conversely, relief and "noncringing") which have been occasioned by the presence or absence of the aversive event. In contrast to the traditionally opposed views of cognitive (S-S) and stimulus response (S-R) learning, our position is that Pavlovian conditioning is more appropriately viewed as involving both S-S learning, pertaining to the general signaling property of the $C S$, and S-R learning, relating to the affective value of the signaled event. A recent distinction by Amsel (1972) between simple, unmediated classical conditioning and Pavlovian preparatory conditioning bears some similarity to our position.

Because the affective value of the signaled event is viewed as a secondary component of the Pavlovian process (and as critically dependent upon such performance factors as US quality, intensity. and magnitude). it should be capable of being rapidly altered or "transmuted" from one value to another without materially affecting the general signaling property of the CS. Thus, were Pavlovian aversive conditioning in one situation to be followed by appetitive discrimination training in another. the general signaling function of the AvCS might readily transfer to the appetitive situation but with its negative affect severely degraded. In effect. a CS could be rapidly transformed from signaling the 
presence or absence of shock to signaling the presence or absence of food. Consequently, the capacity of the specific responses elicited by the CS (e.g. fear) to control performance in the appetitive situation would be reduced. ${ }^{1}$

Recent work of Overmier and Bull (1970) has suggested a similar type of general "cue" function. Studying for the most part the effects of Pavlovian appetitive (Ap) conditioning on instrumental avoidance behavior. these investigators found that an ApCS+ serving as a signal for shock facilitated avoidance learning, and to some extent an ApCS - retarded such learning. These findings, together with other results indicating better conditioning (or less interference in the conditioning) of an ArCS+ when the CS is originally established as an ApCS+ rather than as an ApCS-, have led Overmier and Bull to suggest that once a CS has been associated with one type of reinforcer (e.g.. food), it can easily be switched into a cue for a different type of reinforcer (e.g.. shock).

In a transition from Pavlovian aversive conditioning to appetitive discrimination learning, as in the present research. the general signaling property of the AvCS can be expected to transfer to the appetitive situation but with the result that AvCS+ becomes a signal for the presence of a positive event (the appetitive reinforcer), whereas AvCS - becomes a signal for the absence of the same positive erent. It should be clear that such a change in the affective value of the signal should be accomplished more readily in the context of a nonfearful (i.e.. the no-shock) discrimination arrangement, but retarded or at least obscured in the context of a fearful (i.e., the shock-wrong) discrimination arrangement.

It would be a relatively simple matter to account for the observed facilitation and retardation produced by AvCSt and AvCS - if these CSs were part of the discriminative-stimulus complex; however. in the present research. the CSs were employed as response-contingent events. The present findings are therefore better understood on the basis of the previously elaborated discriminability or distinctive-cue interpretation (Fowler. 1971), but now as applied to the signaling property of the CS rather than to the specific response (e.g.. fear and its feedback) conditioned on the basis of the aversive US. Viewed in relation to S's task of learning to expect food in the correct but not in the incorrect alternative, the administration of an AvCS+ for the correct response should facilitate learning because, as a transmuted signal for the occurrence of the appetitive reinforcer, it can promote S's anticipation of food while in the correct alternative and thus it can function as a signal which increases the discriminability of the stimulus alternatives through the different anticipatory reactions (or "expectancies") that are occasioned in the two alternatives and that are eventually conditioned to the discriminative stimuli. In contrast, an AvCS - for the correct response should retard discrimination learning because. as a transmuted signal for the absence of the appetitive reinforcer, it effectively adds equivalence to the two alternatives; that is, the CS - provides the basis by which $S$ will also anticipate the absence of food in the correct alternative, and hence it reduces discriminability through the similar expectancies that are uccasioned in both the correct and incorrect arms. This interpretation also accounts for the reversal of outcomes when the CSs are administered for the incorrect response (Domber, 1971). In particular, a CSt promotes equivalence by inappropriately signaling the presence of food in the incorrect alternative and thus retards discrimination learning, whereas a $\mathrm{CS}-$ adds discriminability by appropriately signaling that food is absent. and hence it facilitates discrimination learning.

\section{Comparisons with CER and Other Methodologies}

By acknowledging both the signaling and affective components of the Pavlovian process. the present interpretation provides a basis by which the obtained results can be reconciled with the seemingly antithetical outcome of CER and other procedures wherein the effect of an AvCS+. for example, is consistently one of producing suppression rather than facilitation as in the present study. Compared with the present choice methodology. which by its nature is sensitive to the development of differential response probabilities (i.e., the selective acquisition of a response as opposed to its performance. e.g.. speed or rate), the CER methodology focuses on a behavior which has already been acquired and set to a sensitive baseline of performance usually through variable-interval reinforcement. Considering further that Pavlovian aversive training is generally conducted within the same experimental context but typically off baseline (see Davis, 1968), the CER procedure should by its nature be more sensitive to the affective or performance component of Pavlovian conditioning than to a transfer of the signaling property of the CS which is, moreover, administered independently of the measured response, i.e., on a noncontingent basis. Accordingly, the CER procedure can be viewed not as transfering the signaling property of the CS, but rather as opposing those affective processes (cf. "hope" and "fear") and the associated responses that are conditioned on the basis of appetitive and aversive reinforcers.

Our interpretation also has something to add on the traditionally viewed commonality of such affectively positive processes as "hope" and "relief," and similarly of such affectively negative processes as "fear" and "frustration" (cf. McAllister \& McAllister, 1971; Mowrer, 1960: Wagner, 1969). From the present standpoint. such commonality would not be viewed as based on affective components alone (i.e., Av or Ap), but rather on the combination (or product) of the affective and signaling components of the controlling stimulus. So considered. common motivational systems 
may be equally well understood as based on S's hope that food is coming (Ap+) or S's "hope" that shock is not coming $(\mathrm{Av}-)$; and on S's fear that shock is coming $(\mathrm{Avt})$ or S's "fear" that food is not coming (Ap-). (For a similar interpretation. but in the absence of direct supporting data, see Bolles, 1967.)

The present results demonstrate that the components of a Pavlovian CS can be effectively isolated so that the commonality (and hence transferability) of an AvCS+ and an ApCS+ (and similarly of an AvCS- and an ApCS-) relates not to their opposing affective properties, but rather to their common signaling properties. Such an outcome argues for a basic expectancy or cognitive level of functioning in the rat and is not at all at variance with current findings. For example, Trapold and Overmier (1972) have shown that $S$ can learn specific expectancies relating to particular appetitive reinforcers (sucrose solution vs food pellets). Together, their results and ours suggest that the rat may be capable of different levels of cognitive functioning including basic binary or signal-type processing (+ vs --), general affective processing (Ap vs Av), processing of specific types of reinforcer, and even processing of the parameters (e.g.. magnitude) of reinforcement. In this light. our attempts to describe learning and performance effects in terms of the magnitude or the intensity of a particular reinforcer have been quite restricted, to say the least.

\section{REFERENCES}

Amsel, A. Inhibition and mediation in classical, Pavlovian and instrumental conditioning. In R. A. Boakes and M. S. Halliday (Eds.), Inhibition and learning. New York: Academic Press. 1972. Pp. 275-298

Bolles, R. C. Theory of motivation, New York: Harper, 1967.

Davis, H. Conditioned suppression: A survey of the literature. Psychonomic Monograph Supplements, 1968. 2(14, Whole No. 30).

Denny. M. R. Relasation theory and experiments. In F. R. Brush (Ed.), Aversile conditioning and learning. New York: Acadernic Press. 1971. Pp. 235-295.

Domber, E. A.. Ir. Facilitation and retardation of instrumental appetitive learning by prior Pavlovian aversive conditioning. Unpublished PhD thesis, Lniversity of Pittsburgh, 1971

Fago. G.. \& Fowler, H. lacilitated discrimination learning as cffected by response-contingent neutral and aversive stimuli. Learning \& Motivation, 1972, 3, 20-30.

Fowler. H. Iacilitation and inhibition of performance by punishment: The effects of shock intensity and distribution of trials. Journal of Comparative \& Physiological Psychology. 1963. 56, 531-538

Iowler, H. Suppression and tacilitation by response contingent shock. In F. R. Brush (Ed.). Alersive conditioning and learning. New York: Academic Press, 1971. Pp. 537-604.

Fowler, H., Fago, G. C., \& Wischner, G. J, Shock-right facilitation in an easy. noncorrection problem as effected by a delay of reward. Learning \& Motivation, 1971, 2, 235-245.

Fowler, H., Goldman, L., \& Wischner. G. J. Sodium amytal and the shock-right intensity function for visual discrimination learning. Journal of Comparative \& Physiological Psychology. $1968,65,155-159$

Fowler, H., \& Miller, N. E. Facilitation and inhibition of runway performance by hind and fore-paw shock of various intensities. Jcurnal of Comparative \& Physiological Psychology, 1963, 56, 801-805.

Fowler, H., \& Wischner, G. J. The varied functions of punishment in discrimination learning. In B. A. Campbell and R. M. Church (Eds.), Punishment and aversive behavior. Neiv York: Appleton-Century-Crofts, 1969. Pp. 375-420.

Lawrence, D. H. Acquired distinctiveness of cues: I. Transfer between discrimination on the basis of familiarity with the stimulus. Journal of Experimental Psychology, 1949, 39. $770-784$

Lawrence, D. H. Acquired distinctiveness of cues: Il. Selective association in a constant stimulus situation. Journal of Experimental Psychology, 1950, 40, 175-188.

LoLordo, V. M. Positive conditioned reinforcement from aversive situations. Psychological Bulletin, 1969, 72, 193-203.

McAllister, W. R., \& McAllister. D. E. Behavioral measurement of conditioned fear. In F. R. Brush (Ed.), Aversive conditioning and learning. New York: Academic Press. 1971. Pp. 105-179.

Mowrer, O. H. Learning theory and behavior. New York: Wiley, 1960.

Overmier, J. B., \& Bull, J. A., Ill. Influences of appetitive Pavlovian conditioning upon avoidance behavior. In J. $\mathrm{H}$. Reynierse (Ed.), Current issues in animal learning: $A$ colloquium. Lincoln: University of Nebraska Press, 1970. Pp. 117-141.

Rescorla, R. A. Pavlovian conditioning and its proper control procedures. Psychological Review, 1967, 74, 71-80.

Rescorla, R. A. Pavlovian conditioned inhibition. Psychological Bulletin, 1969, 72, 77-94.

Rescorla, R. A., \& Solomon, R. L. Two-process learning theory: Relationships between Pavlovian conditioning and instrumental learning. Psychological Review, 1967, 74, 151-182.

Seligman, M. E. P. Control group and conditioning: A comment on operationism. Psychological Review, 1969, 76, 484-491.

Seligman. M. E. P., Maier, S. F., \& Solomon, R. L. Unpredictable and uncontrollable aversive events. In F. R. Brush (Ed.), Aversive conditioning and learning. New York: Academic Press, 1971. Pp. 347-400.

Trapold. M. A., \& Overmier. J. B. The second learning process in instrumental learning. In A. H. Black and W. F. Prokasy (Eds.). Classical conditioning II: Current research and theory. New York: Appleton-Century-Crofts, 1972. Pp. 427-452.

Wagner. A. R. Frustrative nonreward: A variety of punishment. In B. A. Campbell and R. M. Church (Eds.), Punishment and aversile behavior. New York: A ppleton-Century-Crofts. 1969. Pp. 157-181.

\section{NOTE}

1. Our more recent rescarich on the effect of various US intensities has shown that at the beginning of appetitive discrimination training, running speed in the rewarded arm is suppressed by AvCS+ (and facilitated by $\mathrm{ArCS}-$ ) relative to AvCSo, and moreso the stronger the US intensity, but such effects are relatively short-lived and dissipate within 10-20 CS presentations.

(Received for publication September 28, 1972: accepted October 30.1972 .) 